\title{
Efficacy of vemurafenib in a heavy smoker with BRAF-mutated lung adenocarcinoma: A case report and literature review
}

\author{
XUE LIU $^{1 *}$, XUEFENG FANG $^{1 *}$, HANGUANG HU $^{1}$, XIANHUA FU $^{1}$, YING CHEN $^{2}$ and YING YUAN ${ }^{1}$ \\ Departments of ${ }^{1}$ Medical Oncology and ${ }^{2}$ Radiology, The Second Affiliated Hospital, \\ Zhejiang University School of Medicine, Hangzhou, Zhejiang 310009, P.R. China
}

Received March 29, 2018; Accepted July 30, 2018

DOI: $10.3892 /$ mco.2018.1691

\begin{abstract}
At present, research on BRAF gene mutations appears to be mainly focused on melanoma rather than nonsmall-cell lung cancer (NSCLC). We herein describe the case of a patient with BRAF V600E-mutated advanced NSCLC, whose symptoms were relieved and computed tomography imaging revealed partial response to vemurafenib following failure of chemotherapy. This case demonstrates the promising prospects of BRAF inhibitor treatment in patients with BRAF-mutated NSCLC. Targeted therapies have significantly modified the treatment of NSCLC. However, tumor tissue is frequently hard to obtain, whereas the coincidence rate of gene mutations between the plasma and tumor tissue is 60-80\%. Therefore, in cases where tumor tissue is difficult to obtain, plasma next-generation sequencing may be used to detect gene mutations, which can overcome the limitations of gene detection. Furthermore, due to the tumor heterogeneity, different patients exhibit different gene mutation abundance. Research has demonstrated that mutation abundance is associated with the therapeutic efficacy of epidermal growth factor receptor-tyrosine kinase inhibitors. However, the association between BRAF mutation abundance and the therapeutic effect of BRAF inhibitors requires further verification.
\end{abstract}

\section{Introduction}

The BRAF protein is a member of the RAF-MEK-ERK signal transduction pathway (1). Mutations of BRAF kinase are actively involved in oncogenic proliferation through its constitutive activity (2). Approximately $3 \%$ of non-small-cell lung

Correspondence to: Dr Ying Yuan, Department of Medical Oncology, The Second Affiliated Hospital, Zhejiang University School of Medicine, 88 Jiefang Road, Hangzhou, Zhejiang 310009, P.R. China

E-mail: yuanying1999@zju.edu.cn

${ }^{*}$ Contributed equally

Key words: BRAF mutation, non-small-cell lung cancer, plasma next-generation sequencing, heterogeneity, mutations abundance cancer (NSCLC) cases harbor BRAF mutations (3). However, research on BRAF gene mutations are rarely focused on NSCLC. Targeted therapies have significantly modified the treatment of NSCLC (4), with a large number of targeted therapies for NSCLC already available or currently in clinical trials. However, tumor tissue may be difficult to obtain for gene detection. It has been demonstrated that next-generation sequencing (NGS) tests are superior in terms of sensitivity and specificity compared with non-NGS methods. Additionally, the coincidence rate of gene mutations between the plasma and tumor tissue is $60-80 \%$ (5), suggesting that plasma NGS may be recommended for selection of targeted drugs.

\section{Case report}

In April 2016, a 71-year-old man with a 46-year history of smoking was diagnosed with lung adenocarcinoma of the right middle lobe during a medical examination. A computed tomography $(\mathrm{CT})$ scan revealed a mass in the middle lobe of the right lung with multiple metastatic nodules in both lungs. Pathological assessment confirmed the diagnosis of pulmonary adenocarcinoma. The patient was wild-type for epidermal growth factor receptor (EGFR), Kirsten rat sarcoma viral oncogene homolog (KRAS) and anaplastic lymphoma kinase (ALK).

In May 2016, the patient was treated with carboplatin and pemetrexed (400 and $800 \mathrm{mg} / \mathrm{day}$, respectively) for a total of 6 cycles. A partial response (PR) was achieved. Therefore, in November 2016, the patient was administered pemetrexed maintenance monotherapy $(800 \mathrm{mg} /$ day $)$ for 6 cycles. However, the CT scan after 6 cycles of maintenance therapy revealed progressive disease (PD) indicated by an increase in the size of the lung lesions (Fig. 1). The patient again received chemotherapy with carboplatin and pemetrexed (450 mg twice daily and $800 \mathrm{mg} /$ day, respectively). After 2 cycles of chemotherapy, the appearance of new liver lesions indicated PD. In July 2017, the patient was administered docetaxel (100 mg/day). After 2 cycles of this single-drug chemotherapy, PD was indicated by an increase in the size of the lung lesions and the appearance of new lesions in the pancreas and kidney. The performance status (PS) of the patient quickly deteriorated to 3 , with complaints of abdominal distention and chest pain. In August 2017, plasma NGS analysis revealed a V600E BRAF mutation in exon 15 , with a mutation abundance of 

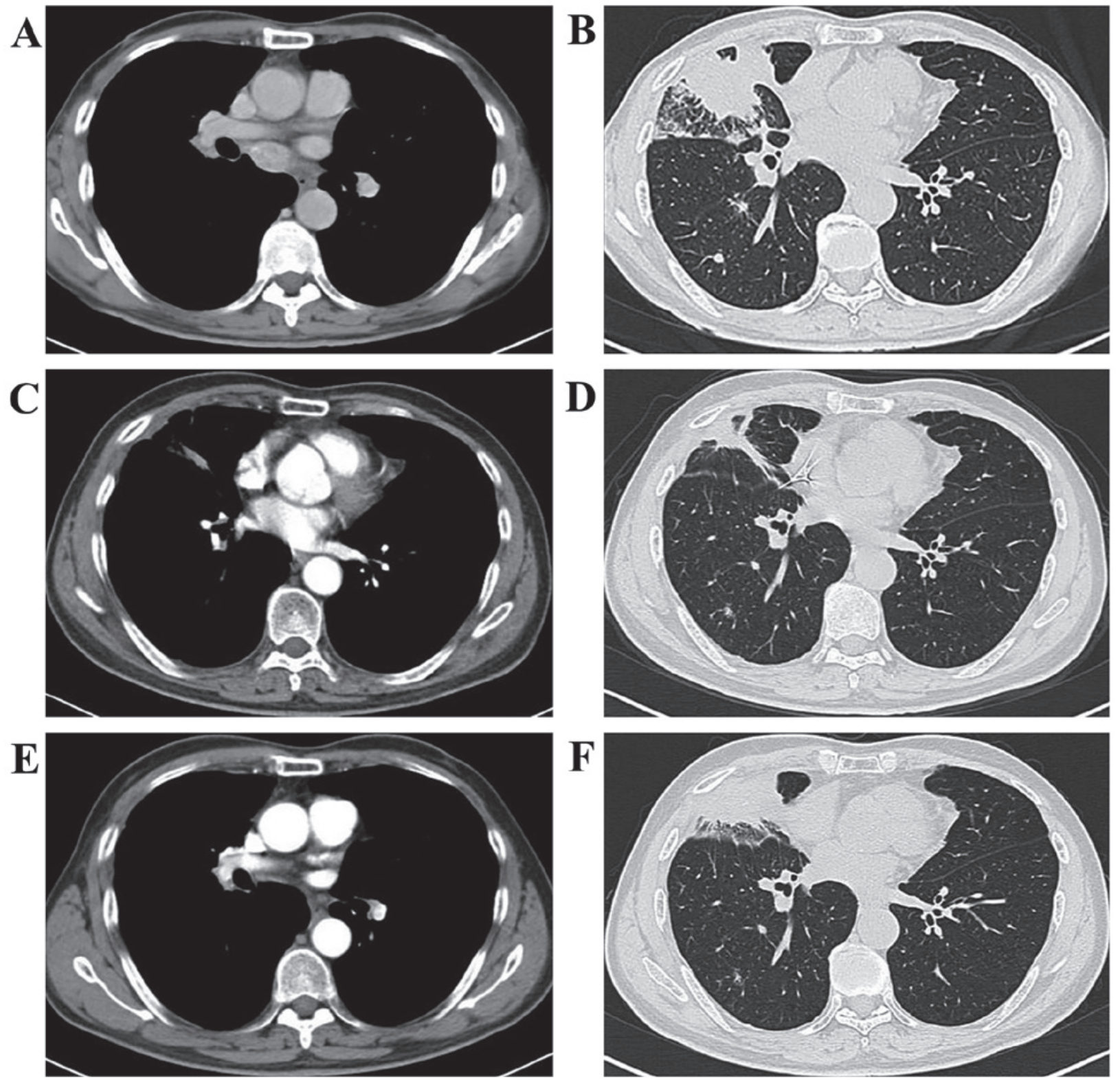

Figure 1. Computed tomography (CT) scans of the present case. (A and B) CT scan prior to treatment. (C and D) CT scan after pemetrexed maintenance monotherapy for 4 cycles. (E and F) CT scan after pemetrexed maintenance for 6 cycles.

$18.62 \%$. Treatment with vemurafenib was initiated at a dose of $720 \mathrm{mg}$ (BID) on August 25, 2017 and the dose was increased to $960 \mathrm{mg}$ from September 1, 2017 to September 5, 2017 to improve the efficacy. However, the vemurafenib dosage was again reduced to $720 \mathrm{mg}$ (BID) due to adverse events such as hand-foot syndrome, liver dysfunction and hypodynamia. The side effects diminished following dosage reduction. After treatment with vemurafenib, the patient's symptoms of abdominal distention and chest pain were ameliorated, and the PS improved to 1. A PR was achieved. However, in December 2017, a CT scan revealed that, although the primary lesion in the lung had shrunk, new liver lesions had appeared, and the treatment efficacy evaluation was again PD (Fig. 2). Furthermore, the PS quickly deteriorated to 3, and the patient again exhibited symptoms of abdominal distension. The patient finally succumbed to the disease on the day of discharge (December 24, 2017), and the cause of death was multiple organ failure. The overall duration of vemurafenib treatment was 3.2 months, and the patient's survival following lung cancer diagnosis was 19.2 months.

\section{Discussion}

Currently, treatments for NSCLC include surgery, chemotherapy, radiotherapy, targeted therapy and immunotherapy. Targeted therapies have significantly changed the treatment of NSCLC (4). However, with regards to the tumor heterogeneity and differences among tissues and organs, the effects of the same targeted agent on tumors located in different areas, even in the same region, may vary greatly $(6,7)$.

BRAF mutations are a contributor to the heterogeneity of lung cancer. The BRAF protein is a member of the RAF-MEK-ERK signal transduction pathway that controls a variety of biological processes (1). Mutations of BRAF kinase are actively involved 

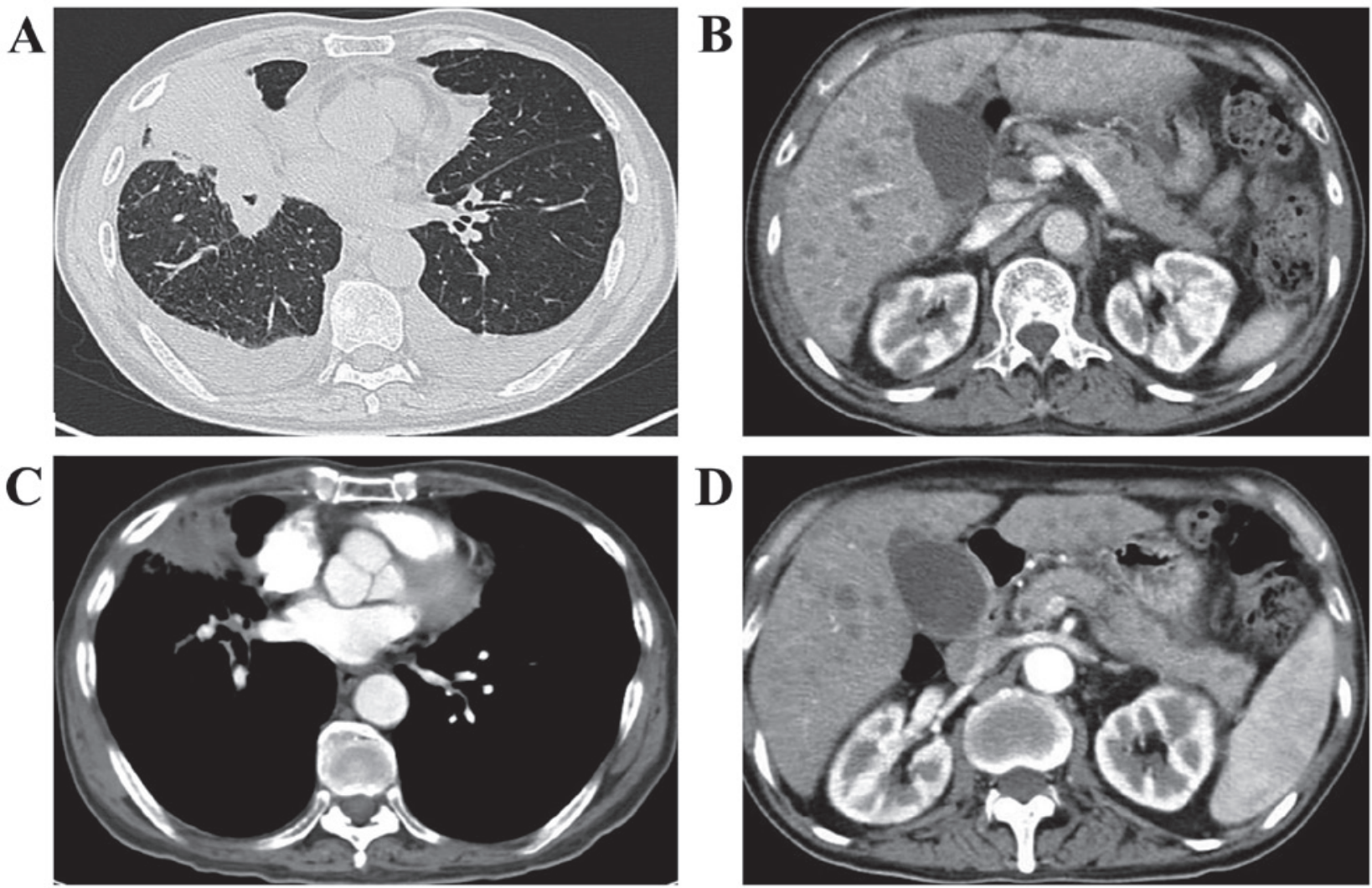

D
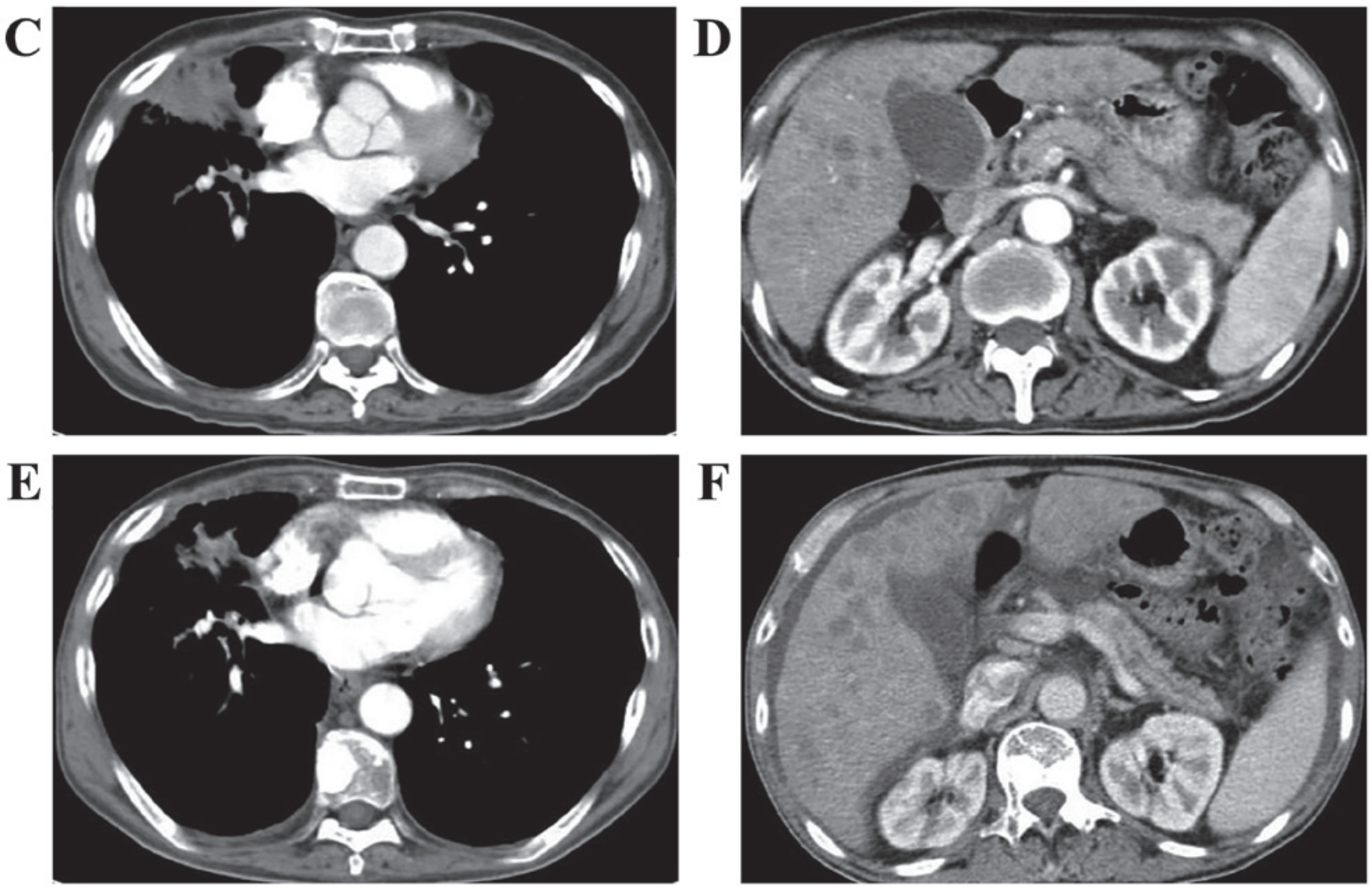

Figure 2. Computed tomography (CT) scans of the present case. (A and B) CT scan prior to vemurafenib treatment. (C and D) CT scan after treatment of vemurafenib for 1 month. (E and F) CT scan after treatment of vemurafenib for 3 months.

in oncogenic proliferation through its constitutive activity (2). BRAF mutations include V600E and non-V600E mutations. Approximately $3 \%$ of NSCLC cases harbor BRAF mutations, which are closely associated with adenocarcinoma (3). The BRAF V600E mutation is more frequent among women and is significantly associated with non-smokers (3). Approximately $55 \%$ of NSCLC patients with a BRAF mutation have been found to harbor the V600E mutation (8). Overall survival (OS) with chemotherapy has improved by the use of vemurafenib (a BRAF inhibitor), which was approved by the Food and Drug Administration and European Medicines Agency for the treatment of metastatic melanoma with a BRAF mutation (9). There are studies and clinical trials showing that BRAF inhibitors are effective against BRAF-mutated NSCLC $(10,11)$. One phase 2 trial included 57 NSCLC cases with the BRAF V600E mutation, and the overall response rate (ORR) was $63.2 \%$, indicating that dabrafenib plus trametinib were promising as novel targeted therapy in BRAF V600E-mutant NSCLC (11). In a histology-independent phase 2 'basket' study of vemurafenib, the response rate of the NSCLC cohort was $42 \%$, and the median progression-free survival (PFS) was 7.3 months (12). In the case described herein, BRAF-mutated NSCLC responded to vemurafenib treatment.

In the present case, the dose of vemurafenib was reduced due to adverse events, including increased levels of total bilirubin and PS 3. The most common adverse reactions associated with vemurafenib include diarrhea, fever, rash, photosensitivity, hand-foot syndrome, joint pain, abnormal liver function and QT interval prolongation. In Chinese studies, the adverse reactions of vemurafenib were mainly grade $1-2$, whereas events of grade $\geq 3$ were rarely reported. The incidence of melanotic nevus was high, but there are no reports of melanotic nevus progression, and the use of vemurafenib in cutaneous squamous cell carcinoma has not yet been reported. In other studies, the adverse 
reactions of vemurafenib were mainly grade $1-2$ with grade $\geq 3$ reactions rarely reported; the majority of the grade 3 reactions were observed in cutaneous squamous cell carcinoma.

There is currently a great number of targeted therapies for NSCLC that are already available or undergoing evaluation in clinical trials. Therefore, molecular characterization of tumors using NGS technology has become a valuable tool that aids treatment decision-making and the clinical management of NSCLC patients (13). Non-NGS testing includes a number of tests for alterations of 11 genes known to be involved in lung cancer $(14,15)$. All four types of DNA alterations in cancer may be diagnosed though hybrid capture-based NGS platforms with better sensitivity and specificity compared with non-NGS tests (13). One study indicated that hybrid capturebased NGS identified actionable genomic alterations in $65 \%$ of tumors with genomic alterations that were not detected by more dated extensive non-NGS testing (14). Furthermore, dysregulated miRNA profiles may be specifically identified using NGS technology in resectable NSCLC, which may be a potential predictor of recurrence-free survival and OS (16).

At present, plasma NGS technology is applied in clinical practice. Sensitive polymerase chain reaction analysis techniques and high-throughput NGS technologies have been developed to perform genetic analyses of circulating free DNA. The coincidence rate of gene mutations between the plasma and tumor tissue is $60-80 \%$ (5). Therefore, in cases where tumor tissue is difficult to obtain, plasma NGS may be used to detect gene mutations. Limitations of gene detection in tumor tissues are overcome by plasma NGS technology. The high specificity of NGS may be directly recommended for the selection of targeted drugs based on the results of the plasma DNA analysis (17).

Due to the tumor heterogeneity, different patients may harbor different gene mutations, such as the multiple mutations of EGFR. The mutation abundance is associated with the therapeutic effect of EGFR-tyrosine kinase inhibitors. Patients with EGFR mutations had a significantly longer PFS compared with those without EGFR mutations (18). Patients with a high level of EGFR mutation abundance exhibited better response to treatment compared with patients in the low-level group (19). However, there are no relevant studies on the abundance of BRAF mutations. It remains unknown whether BRAF mutation abundance affects the response of patients to targeted drug therapy to the same extent as EGRF mutation abundance, and further experiments are required to elucidate this hypothesis.

In conclusion, we herein described the case of a heavy smoker with BRAF-mutated NSCLC. The patient was wildtype for EGFR and ALK. Following failure of the second-line treatment, the patient was administered vemurafenib when the NGS analysis revealed a V600E BRAF mutation in exon 15 and a mutation abundance of $18.62 \%$, after which time the CT scan revealed a PR. However, in December 2017, the CT scan revealed PD (PFS of 3.2 months). This case provides an example of vemurafenib administration based on NGS, and highlights the potential value of vemurafenib in the treatment of advanced lung cancer harboring a BRAF mutation.

\section{Acknowledgements}

Not applicable.

\section{Funding}

The present study was supported by the National Natural Science Foundation of China (grant no. 81500012), the Zhejiang Provincial Natural Science Foundation of China (grant no. LQ16H010001) and the Medical and Health Technology Program of Zhejiang Province (grant nos. 2015111464 and 2017204226).

\section{Availability of data and materials}

Not applicable.

\section{Authors' contributions}

YY contributed to the patient's treatment plan. XL and XF collected and interpreted the data. HH, XF and YC contributed to the management of patient. All the authors read and approved the final version of the manuscript.

\section{Ethics approval and consent to participate}

Not applicable.

\section{Patient consent for publication}

The patient's family consent to the publication of the case details and associated images.

\section{Competing interests}

The authors declare that they have no competing interests.

\section{References}

1. Peyssonnaux $\mathrm{C}$ nd Eychène A: The Raf/MEK/ERK pathway: New concepts of activation. Biol Cell 93: 53-62, 2001.

2. Huebner C, Weber R and Lloydd R: A HRM assay for identification of low level BRAF V600E and V600K mutations using the CADMA principle in FFPE specimens. Pathology 49: 776-783, 2017.

3. Chen D, Zhang LQ, Huang JF, Liu K, Chuai ZR, Yang Z, Wang YX, Shi DC, Liu Q, Huang Q, et al: BRAF mutations in patients with non-small cell lung cancer: A systematic review and meta-analysis. PLoS One 9: e101354, 2014.

4. Tamiya M, Tamiya A, Shiroyama T, Takeoka S, Naito Y, Omachi N, Kimura Y, Morishita N, Suzuki H, Okamoto N, et al: Phasel study of cisplatin plus pemetrexed with erlotinib and bevacizumab for chemotherapy-naïve advanced non-squamous non-small cell lung cancer with EGFR mutations. Invest New Drugs: Nov 4, 2017 (Epub ahead of print).

5. Oxnard GR, Paweletz CP and Sholl LM: Genomic analysis of plasma cell-free DNA in patients With Cancer. JAMA Oncol 3: 740-741, 2017.

6. Grzywa TM, Paskal W and Włodarski PK: Intratumor and intertumor heterogeneity in melanoma. Transl Oncol 10: 956-975, 2017.

7. Martelotto LG, Ng CK, Piscuoglio S, Weigelt B and Reis-Filho JS: Breast cancer intra-tumor heterogeneity. Breast Cancer Res 16: 210, 2014.

8. Villaruz LC, Socinski MA, Abberbock S, Berry LD, Johnson BE, Kwiatkowski DJ, Iafrate AJ, Varella-Garcia M, Franklin WA, Camidge DR, et al: Clinicopathologic features and outcomes of patients with lung adenocarcinomas harboring BRAF mutations in the Lung Cancer Mutation Consortium. Cancer 121: 448-456, 2015.

9. Czirbesz K, Gorka E, Balatoni T, Pánczél G, Melegh K, Kovács P, Gézsi A and Liszkay G: Efficacy of vemurafenib treatment in 43 metastatic melanoma patients with BRAF mutation. Single-institute retrospective analysis, early real-life survival data. Pathol Oncol Res: Sep 29, 2017 (Epub ahead of print). 
10. Noeparast A, Teugels E, Giron P, Verschelden G, De Brakeleer S, Decoster L and De Grève J: Non-V600 BRAF mutations recurrently found in lung cancer predict sensitivity to the combination of Trametinib and Dabrafenib. Oncotarget 8: 60094-60108, 2016.

11. Planchard D, Besse B, Groen HJM, Souquet PJ, Quoix E, Baik CS, Barlesi F, Kim TM, Mazieres J, Novello S, et al: Dabrafenib plus trametinib in patients with previously treated BRAF(V600E)-mutant metastatic non-small cell lung cancer: An open-label, multicentre phase 2 trial. Lancet Oncol 17: 984-993, 2016

12. Hyman DM, Puzanov I, Subbiah V, Faris JE, Chau I, Blay JY, Wolf J, Raje NS, Diamond EL, Hollebecque A, et al: Vemurafenib in Multiple Nonmelanoma Cancers with BRAF V600 Mutations. N Engl J Med 373: 726-736, 2015.

13. Tsoulos N, Papadopoulou E, Metaxa-Mariatou V, Tsaousis G, Efstathiadou C, Tounta G, Scapeti A, Bourkoula E, Zarogoulidis P, Pentheroudakis G, et al: Tumor molecular profiling of NSCLC patients using next generation sequencing. Oncol Rep 38: 3419-3429, 2017

14. Drilon A, Wang L, Arcila ME, Balasubramanian S, Greenbowe JR, Ross JS, Stephens P, Lipson D, Miller VA, Kris MG, et al: Broad, Hybrid Capture-Based Next-Generation Sequencing Identifies Actionable Genomic Alterations in Lung Adenocarcinomas Otherwise Negative for Such Alterations by Other Genomic Testing Approaches. Clin Cancer Res 21: 3631-3639, 2015.

15. Chaft JE, Arcila ME, Paik PK, Lau C, Riely GJ, Pietanza MC, Zakowski MF, Rusch V, Sima CS, Ladanyi M, et al: Coexistence of PIK3CA and other oncogene mutations in lung adenocarcinoma-rationale for comprehensive mutation profiling. Mol Cancer Ther 11: 485-491, 2012.
16. Suh JH, Johnson A, Albacker L, Wang K, Chmielecki J Frampton G, Gay L, Elvin JA, Vergilio JA, Ali S, et al; Comprehensive Genomic Profiling Facilitates Implementation of the National Comprehensive Cancer Network Guidelines for Lung Cancer Biomarker Testing and Identifies Patients Who May Benefit From Enrollment in Mechanism-Driven Clinical Trials: Comprehensive genomic profiling facilitates implementation of the national comprehensive cancer network guidelines for lung cancer biomarker testing and identifies patients who may benefit from enrollment in mechanism-driven clinical trials. Oncologist 21: 684-691, 2016.

17. Xiong L, Cui S, Ding J, Sun Y, Zhang L, Zhao Y, Gu A, Chu T, Wang $\mathrm{H}$, Zhong $\mathrm{H}$, et al: Dynamics of EGFR mutations in plasma recapitulates the clinical response to EGFR-TKIs in NSCLC patients. Oncotarget 8: 63846-63856, 2017.

18. Shan L, Wang Z, Guo L, Sun H, Qiu T, Ling Y, Li W, Li L, Liu X, Zheng B, et al: Concurrence of EGFR amplification and sensitizing mutations indicate a better survival benefit from EGFR-TKI therapy in lung adenocarcinoma patients. Lung Cancer 89: 337-342, 2015.

19. Li H, Wang Y, Su F, Li J and Gong P: Monitoring of cyclooxygenase-2 levels can predict EGFR mutations and the efficacy of EGFR-TKI in patients with lung adenocarcinoma. Int J Clin Exp Pathol 8: 5577-5583, 2015. 